\title{
Coronal Mass Ejections and Space Weather
}

\author{
David F. Webb ${ }^{1,2}$ \\ ${ }^{1}$ Inst. for Scientific Research, Boston College, 140 Commonwealth Ave., Chestnut Hill, MA \\ 02467, USA email: david.webb@hanscom.af.mil \\ ${ }^{2}$ Also at: Space Vehicles Directorate, Air Force Research Lab, Hanscom Air Force Base, MA \\ 01731-3010 USA
}

\begin{abstract}
Coronal mass ejections (CMEs) are a key aspect of coronal and interplanetary dynamics. They can inject large amounts of mass and magnetic fields into the heliosphere, causing major geomagnetic storms and interplanetary shocks, a key source of solar energetic particles. Studies over the past decade using the excellent data sets from the SOHO, TRACE, Wind, $\mathrm{ACE}$ and other spacecraft and ground-based instruments have improved our knowledge of the origins and early development of CMEs at the Sun and how they affect space weather at Earth. I review some key coronal properties of CMEs, their source regions, their manifestations in the solar wind, and their geoeffectiveness. Halo-like CMEs are of special interest for space weather because they suggest the launch of a geoeffective disturbance toward Earth. However, their correspondence to geomagnetic storms varies over the solar cycle. Although CMEs are involved with the largest storms at all phases of the cycle, recurrent features such as interaction regions and high speed wind streams can also be geoeffective. A new heliospheric experiment, the Solar Mass Ejection Imager, has completed its first year in orbit and I give some early results.
\end{abstract}

\section{Solar and Geomagnetic Activity Over the Solar Cycle}

It well known that the level of geomagnetic activity tends to follow the solar sunspot cycle. Sunspot counts are a useful measure of the general level of magnetic activity emerging through the photosphere of the Sun, and they rise and fall relatively uniformly over the cycle. Other major classes of solar activity also tend to track the sunspot number during the cycle, including active regions, flares, filaments, and coronal mass ejections (CMEs) (e.g., Webb and Howard, 1994). This activity is transmitted to Earth through the solar corona and its expansion into the heliosphere as the solar wind.

The cyclical variation of the solar magnetic field can be summarized as follows. The sunspot number indicates the scale of the emergence through the solar surface of regions of the strongest flux. The photospheric flux is indicative of the strength of the toroidal flux which in a Babcock-type dynamo model of solar activity is built up from the basic solar dipole field over the solar cycle. The total photospheric flux varies by about a factor of 3-10 over the cycle. A useful technique for estimating the interplanetary flux is to calculate the flux through the source surface above which the field is assumed to be radial. The source surface flux tracks the lower order, dipole contribution of the solar field over the cycle and is indicative of the variation of the total open flux from the Sun. Although the magnitude of the total interplanetary magnetic field (IMF) at $1 \mathrm{AU}$ is stronger at maximum and weaker near minimum than the total open flux (e.g., Wang et al., 2000), it is still strong during the declining phase when it is dominated by the open flux in high speed wind streams from coronal holes.

Since solar activity is transmitted to Earth via the solar wind, how closely does the cycle of geoactivity follow that of solar activity? The overall correspondence between the sunspot and geoactivity cycles can be seen in long-term plots comparing various indices of the two kinds of activity. On an annual basis the geoactivity cycle has more structure 
than the solar cycle. Figure 1 shows that the geomagnetic index $A p$ is more variable than sunspot number, but does tend to track the sunspot cycles in amplitude.

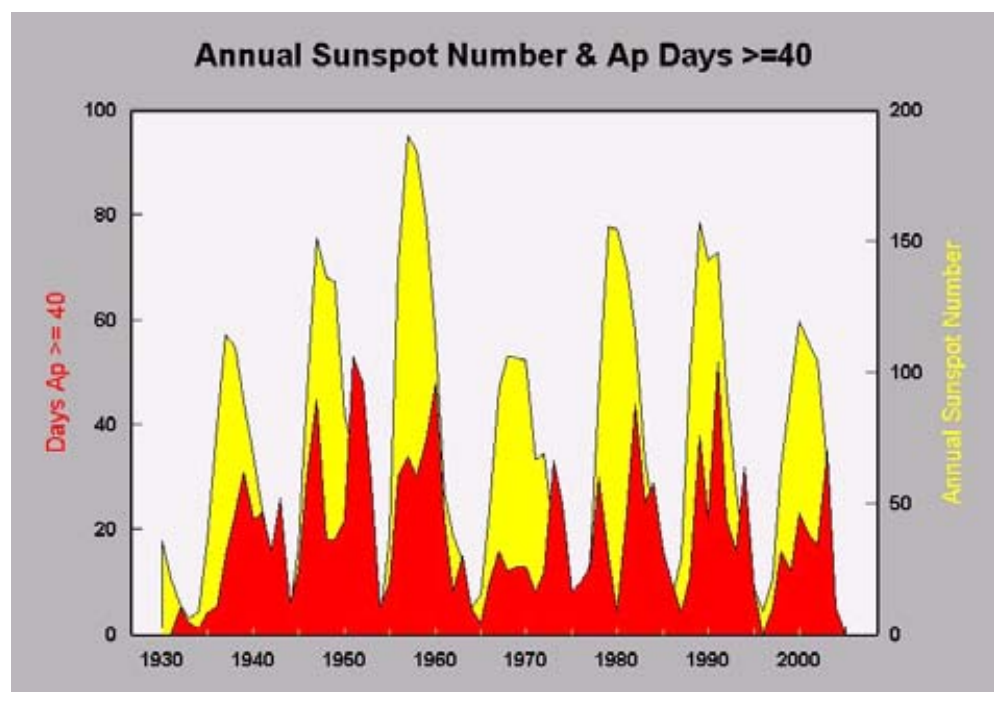

Figure 1. Annual number of geomagnetic disturbed days with Ap index $>40$ (black or red area) vs. annual sunspot number (light gray or yellow area) for solar cycles 17-23. Courtesy NOAA National Geophysical Data Center, Boulder, CO.

Figure 1 also illustrates the double-peaked nature of the geoactivity cycle. In general, geomagnetic activity exhibits a peak near sunspot maximum and another during the declining phase of the cycle. These peaks vary in amplitude and timing, and the peak around maximum may be multiple (Richardson et al., 2000). The two main peaks represent the maximum phases of two components of geoactivity with different solar and heliospheric sources. The first peak is associated with transient solar activity, i.e., CMEs, that tracks the solar cycle in amplitude and phase. The later peak is attributed to recurrent high speed streams from coronal holes, and is often higher than the early peak.

Richardson et al. (2000; 2001) have studied the relative contributions of different types of solar wind structures to the aa index from 1972-1986. They identified CME-related flows, corotating high-speed streams, and slow flows near the Earth, finding that each type contributed significantly to $a a$ at all phases of the cycle. For example, CMEs contribute $\sim 50 \%$ of $a a$ at solar maximum and $\sim 10 \%$ outside of maximum, and high speed streams contribute $\sim 70 \%$ outside of maximum and $\sim 30 \%$ at maximum. Thus, both types of sources, CMEs and coronal holes/high speed streams, contribute to geoactivity all phases of the cycle. CMEs, however, are responsible for the most geoeffective solar wind disturbances and, therefore, the largest storms.

\section{Properties and Solar Signatures of CMEs}

CMEs consist of large structures containing plasma and magnetic fields that are expelled from the Sun into the heliosphere. The plasma observed in a CME is entrained on expanding magnetic field lines, which can have the form of helical field lines with changing pitch angles, i.e., a flux rope. This paper reviews the well-determined coronal properties of CMEs and what we know about their source regions, and some key signatures of CMEs in the solar wind. I emphasize observations of halo CMEs, as observed by the SOHO LASCO coronagraphs, because they are important for space weather studies. 
Most observations of CMEs have been made by white light coronagraphs, operating in space. These observations are based on the Thomson scattering process, wherein dense plasma structures in the corona become visible via photospheric light scattered off of the free electrons in the plasma. The LASCO coronagraphs (Brueckner et al. 1995) provide the most recent and most extensive spaceborne white light observations of CMEs. These data have been acquired since early 1996, providing fairly continuous coverage of the corona and CMEs from the solar activity minimum through the decline of cycle 23 . The LASCO observations of CMEs are complemented by other SOHO instruments operating at coronal wavelengths, especially EIT, UVCS and CDS, and the Yohkoh and TRACE spacecraft (see Hudson \& Cliver, 2001).

The measured properties of CMEs include their occurrence rates, locations relative to the solar disk, angular widths, speeds, and masses and energies (e.g., Kahler, 1992; St. Cyr et al., 2000; Webb, 2002; Gopalswamy et al., 2003; Yashiro et al., 2004). There is a large range in the basic properties of CMEs. Their speeds, accelerations, masses and energies extend over 2-3 orders of magnitude (e.g., Vourlidas et al., 2002), and their widths exceed by factors of 3-10 the sizes of flaring active regions.

CMEs can exhibit a variety of forms, some having the classical "three-part" structure and others being more complex with interiors with bright emitting material. The basic structure of the former kind consists of a bright leading arc followed by a darker, lowdensity cavity and a bright core of denser material. Many different forms of CMEs have been observed, including those with large circular regions resembling flux ropes and halo CMEs.

Because of their increased sensitivity, field of view and dynamic range, the SOHO LASCO coronagraphs now frequently observe halo CMEs, which appear as expanding, circular brightenings that completely surround the coronagraphs' occulting disks (Howard et al., 1982). This suggests that these are 'normal' CMEs seen in projection (Burkepile et al., 2004) to be moving outward either toward or away from the Earth. Observations of associated activity on the solar disk are necessary to distinguish whether a halo CME was launched from the front or backside of the Sun. CMEs which have a larger apparent angular size than typical limb CMEs but do not appear as complete halos, are called 'partial halo' CMEs. Halo CMEs are important for three reasons: 1) They are the key link between solar eruptions and many space weather phenomena such as major storms and solar energetic particle events; 2) The source regions of frontside halo CMEs are usually located within a few tens of degrees of Sun center, as viewed from Earth (Cane et al., 2000; Webb 2002). Thus, these regions can be studied in greater detail than for most CMEs which are observed near the limb: 3) Frontside halo CMEs must travel approximately along the Sun-Earth line, so their internal material can be sampled in situ by spacecraft near the Earth. Three spacecraft, SOHO, Wind and ACE, now provide solar wind measurements upstream of Earth.

The frequency of occurrence of CMEs observed in white light tends to track the solar cycle in both phase and amplitude, which varies by an order of magnitude over the cycle (Webb and Howard, 1994). LASCO has now observed from solar minimum in early 1996 through the declining phase of the current (23rd) solar cycle. It has detected CMEs at a rate slightly higher than earlier observations, reaching $\sim 5$ /day at maximum (St. Cyr et al., 2000; Gopalswamy et al., 2003). Halo CMEs occur at a rate of about $10 \%$ that of all CMEs, but full halo CMEs are only detected at a rate of $\sim 5 \%$ of all CMEs. If CMEs occurred randomly at all longitudes and LASCO detected all of them, this rate should be about 15\%, suggesting that LASCO sees as halos CMEs that are brighter (i.e., denser) than average. In turn this implies that LASCO may miss fainter CMEs near sun center. 
In support, Tripathi et al. (2004) found that all (8\%) post- eruptive EIT arcades not associated with LASCO CMEs occurred within $40^{\circ}$ of sun center.

The latitude distribution of the central position angles of CMEs tends to cluster about the equator at minimum but broaden over all latitudes near solar maximum. Hundhausen (1993) noted that this CME latitude variation more closely parallels that of streamers and prominences than of active regions, flares or sunspots. On the contrary, with previous coronagraphs the angular size distribution of CMEs seemed to vary little over the cycle, maintaining an average width of about $45^{\circ}$ (SMM-Hundhausen, 1993; Solwind-Howard et al., 1985). However, the CME size distribution observed by LASCO is affected by its increased detection of very wide CMEs, especially halos. Including halo CMEs from Jan. 1996--June 1998, St. Cyr et al. (2000) found the average (median) width of LASCO CMEs was $72^{\circ}\left(50^{\circ}\right)$. Including all measured LASCO CMEs through 2002 of $20-120^{\circ}$ in width, Yashiro et al. (2004) found the average widths to vary, from $47^{\circ}$ at minimum to $61^{\circ}$ at maximum (1999), then declining again.

Estimates of the apparent speeds of the leading edges of CMEs range from about 20 to over $2500 \mathrm{~km} \mathrm{~s}^{-1}$, or from well below the sound speed in the lower corona to well above the Alfven speed. The annual average speeds of SOLWIND and SMM CMEs varied over the solar cycle from about $150-475 \mathrm{~km} \mathrm{~s}^{-1}$, but their relationship to sunspot number was unclear (Howard et al., 1986; Hundhausen et al., 1994). However, LASCO CME speeds do show a tendency to increase with sunspot number in this cycle (St. Cyr et al., 2000; N. Gopalswamy et al., 2003; Yashiro et al. (2004), from $280 \mathrm{~km} \mathrm{~s}^{-1}$ to $\sim 500 \mathrm{~km} \mathrm{~s}^{-1}$ near maximum. The annual average speed of full halo CMEs is 1.5-2 times greater than that of all CMEs (Webb, 2002; Yashiro et al., 2004), suggesting that LASCO sees as halos CMEs which are faster and, hence, more energetic than the average CME. Above a height of about $2 \mathrm{R}_{S}$ the speeds of typical CMEs are relatively constant, although the slowest CMEs tend to show acceleration while the fastest CMEs decelerate (St. Cyr et al., 2000; Yashiro et al., 2004). Clearly the acceleration for most CMEs occurs low in the corona.

Finally, the masses and energies of CMEs require difficult instrument calibrations and have large uncertainties. The average mass of CMEs derived from the older coronagraph data (Skylab, SMM and Solwind) have now been confirmed by LASCO measurements (Vourlidas et al., 2002) to be a few $\times 10^{15}$ g. Studies using Helios (Webb et al., 1996) and LASCO (Vourilidas et al., 2000) data indicate that older CME masses may have been underestimates because mass outflow can continue well after the CME's leading edge leaves the instrument field of view. Average CME kinetic energies from all the data sets are consistent at a few $\times 10^{30} \mathrm{erg}$. The CME kinetic energy distribution has a a power law index of $\sim-1$ (Vourlidas et al., 2002), different from that of flares ( -2; Hudson 1990).

Next I briefly summarize our knowledge of the near-surface features that appear associated with the initiation of CMEs (see recent reviews, e.g., Webb, 2002; Cliver \& Hudson, 2002; Gopalswamy, 2004). Many CMEs appear to arise from large-scale, closed structures, particularly preexisting coronal streamers (e.g., Hundhausen, 1993). Many energetic CMEs actually involve the disruption ("blowout") of a preexisting streamer, which can increase in brightness and size for days before erupting as a CME (Howard et al., 1985; Hundhausen, 1993).

Previous statistical association studies indicated that erupting filaments and X-ray events, especially of long duration, were the most common near-surface activity associated with CMEs. Most optical flares occur independently of CMEs and even those accompanying CMEs may be a consequence rather than a cause of CMEs. The fastest, most energetic CMEs, however, are usually also associated with surface flares, and reported flares are associated with most ( 85\%) frontside, full halo CMEs (e.g., Webb, 2002). 
This rate may be higher than reported previously because the sources of halo CMEs can be clearly viewed near sun center.

Comparisons of low coronal soft X-ray and EUV data with the white light observations have provided many insights into the source regions of CMEs. Sheeley et al. (1983) showed that the probability of associating a CME with a soft X-ray flare increased linearly with the flare duration, reaching $100 \%$ for flare events of duration $>6$ hours. The SMM CME observations indicated that the estimated departure time of flare-associated CMEs typically preceded the flare onsets. Harrison (1986) found that such CMEs were initiated during weaker soft X-ray bursts that preceded any subsequent main flare by tens of minutes, and that the main flares were often offset to one side of the CME. The latitude distribution of LASCO CMEs peaks at the equator, but the distribution of EIT EUV activity associated with these CMEs is bimodal with peaks $30^{\circ}$ north and south of the equator (Plunkett et al., 2002). This offset is confirmed for the distribution of sources associated with halo CMEs (Webb 2002). This pattern indicates that many CMEs can involve more complex, multiple polarity systems (Webb et al., 1997) such as modeled by Antiochos et al. (1999).

The most obvious coronal signatures of CMEs in the low corona are the arcades of bright loops which develop after the CME material has apparently left the surface (Kahler, 1977-Skylab; Hudson \& Webb, 1997-Yohkoh; Tripathi et al., 2004-EIT). Prior to the eruption, an S-shaped structure called a sigmoid can develop, sometimes in association with a filament activation. A sigmoid is indicative of a highly sheared, non-potential coronal magnetic field, and might be an important precursor of a CME (e.g., Canfield et al. 1999), although its geoeffectiveness has not been established. Eventually an eruptive flare can occur, resulting in the bright, long-duration arcade of loops. Sterling et al. (2000) call this process "sigmoid-to-arcade" evolution. These arcades suggest the eruption and subsequent reconnection of strong magnetic field lines associated with the CME system. Tripathi et al. (2004) find that nearly all (92\%) of EIT post- eruptive arcades from 19972002 were associated with LASCO CMEs. Dimming regions observed in X-rays and in the EUV imply that material is evacuated from the low corona (c.f., Hudson \& Webb, 1997). The dimming regions can be much more extensive than the flaring activity and often map out the apparent base of the white light CME (Thompson et al., 2000). Thus, the dimming events appear to be one of the earliest and best-defined signatures in soft X-ray and EUV emission of the actual mass ejected from the low corona.

Surveys of solar activity associated with frontside halo CMEs have been made primarily with low coronal images from the EIT and Yohkoh SXT instruments. The activity associated with halo CMEs includes the formation of dimming regions, of long-lived loop arcades, flaring active regions, large-scale coronal waves that propagate outward from the CME source region, and filament eruptions. Specifically, Webb (2002) finds that $\frac{2}{3}$ of the halo CMEs are associated with filament eruptions and with dimmings. The frequent detection of coronal EUV waves was an exciting discovery of the EIT observations. Although Biesecker et al. (2002) found a CME associated with nearly every EIT wave, apparently not all CMEs are associated with waves since only about half of all halo CMEs have EIT waves (e.g., Webb 2002).

\section{CMEs in the Heliosphere}

\subsection{Signatures of ICMEs}

CMEs carry into the heliosphere large amounts of coronal magnetic fields and plasma, which can be detected by remote sensing and in-situ spacecraft observations. The passage 
of this material past a single spacecraft is marked by distinctive signatures, but with a great degree of variation from event to event (e.g., Gosling, 1993). These signatures include transient interplanetary shocks, depressed proton temperatures, cosmic ray depressions, flows with enhanced helium abundances, unusual compositions of ions and elements, and magnetic field structures consistent with looplike topologies.

A widely used single-parameter signature of ejecta is the occurrence of counterstreaming suprathermal electrons. Since suprathermal electrons carry electron heat flux away from the Sun along magnetic field lines, when found streaming in both directions along the field they are interpreted as signatures of closed field lines and, thus, as a good proxy for CMEs in the solar wind (e.g., Gosling, 1993). An important multiple signature of an interplanetary (I)CME, is a magnetic cloud, defined as several-hour flows with large-scale rotations of unusually strong magnetic field accompanied by low ion temperatures. The magnetic field data from clouds often provide good fits to flux rope models.

Another class of ejecta plasma signatures are the abundances and charge state compositions of elements and ions which are systematically different in ICMEs compared with other kinds of solar wind (von Steiger \& Zurbuchen, 2003). As the corona expands outward, the electron density decreases so rapidly that the plasma becomes collisionless and the relative ionization states become constant, thus reflecting the conditions of origin in the corona. The charge states of minor ions in CME flows usually suggest slightly hotter than normal coronal conditions (i.e., $>2 \mathrm{MK}$ ) at this "freezing in" location. In addition, transient flows often exhibit element and ion abundances that are enhanced relative to the typical solar wind. Unusually low ionization states of He and minor ions have also been detected in CME flows. Although rarely observed before, enhanced $\mathrm{He}^{+}$flows have been detected in the flows from several recent halo CMEs with more sensitive instruments on the SOHO, Wind and ACE spacecraft. In each of these events an erupting filament-halo CME could be associated with either a dense and compact 'plug' or an extended flow of cool plasma in the trailing edge of a magnetic cloud. This is likely material from the filament itself, consistent with near-Sun observations showing that erupting filaments lag well behind the leading edge of their associated CMEs.

\subsection{Remote Sensing of CMEs}

Several techniques have been developed to remotely detect and track disturbances related to CMEs in the interplanetary medium (Jackson, 1992). These have utilized radio and white light wavelengths to detect and crudely image these structures. The radio techniques are kilometric radio observations from space and interplanetary scintillation (IPS) observations from the ground. The kilometric observations can track the emission typically from strong shocks traveling ahead of fast CMEs. Such instruments have been flown on the ISEE-3, Wind and Ulysses spacecraft, and will be on the twin STEREO spacecraft to be launched in 2006. The IPS technique relies on measurements of the fluctuating intensity level of strong, point-like distant radio sources from one or more ground arrays operating in the $\mathrm{MHz}$ range. IPS arrays detect changes in density inhomogeneties in the (local) interplanetary medium moving across the line of sight to the source. Disturbances are detected by either an enhancement of the scintillation level and/or an increase in velocity. The technique suffers from relatively poor temporal $(\sim 24$ hour $)$ and spatial (few bright sources) resolution, ionospheric noise which limits viewing near the Sun and near the horizon, and a model-dependence for interpreting the signal as density or mass.

Until recently, the only heliospheric observations in white light of ICMEs had been made by the zodiacal light photometers on the twin Helios spacecraft which orbited the Sun in the 1970s and early 1980s. On each spacecraft 3 sensitive telescopes swept the celestial sphere at 16,31 and $90^{\circ}$ ecliptic latitude. Contour images could be constructed 

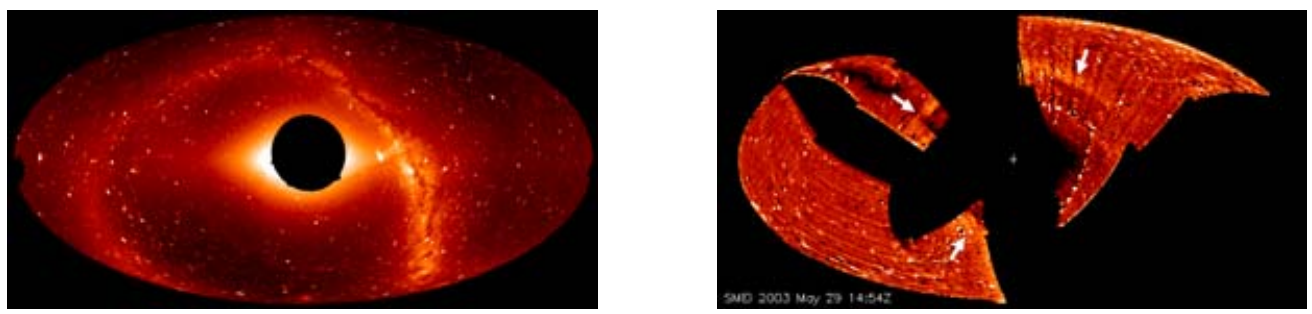

Figure 2. All-sky images from SMEI. Left) Composite all-sky image taken in Feb. 2003. An equal-area Hammer-Aitoff projection centered on the Sun with the North and South ecliptic poles at top and bottom, resp. The dark circle is a zone of exclusion $20^{\circ}$ in radius centered on the Sun. Right) Earthward halo CME seen from SMEI on May 29, 2003. The halo was visible as an arc over $>150^{\circ}$ of sky (arrows). In addition to the exclusion circle, other blacked-out areas are due to shuttering of bright sunlight and noise (CCD "hits") from particles in Coriolis' 840-km circular Earth orbit.

with several hour time resolution but with only crude spatial resolution. Like solar coronagraphs, Helios detected ICMEs by the Thomson scattering process.

The Helios photometers provided the heritage for an instrument called the Solar Mass Ejection Imager (SMEI) developed as a proof-of-concept experiment for operational forecasting purposes. The experiment (Eyles et al., 2003) was designed, constructed and is now operated by a team from the U.S. Air Force Research Laboratory, the University of California at San Diego, the University of Birmingham in England, and Boston College. Launched in January 2003 on the Coriolis spacecraft, SMEI images nearly the entire sky in white light once per spacecraft orbit, using three baffled camera systems with CCD detectors. Each camera frame covers a different $3 \times 60^{\circ}$ slice of the sky. When these frames are stitched together and mapped into ecliptic coordinates, a nearly complete sky map is constructed for an orbit. Figure 2a shows an early "first-light" image of the full sky from SMEI.

During its first year SMEI observed over 100 CMEs, most viewed in projection coming off the solar limb. SMEI has accomplished its major proof-of-concept milestone by detecting and tracking several Earth-directed ("halo") CMEs. The first was observed in late May 2003 and is shown in Fig.2b (Tappin et al., 2004). This fast $\left(\sim 1000 \mathrm{~km} \mathrm{~s}^{-1}\right)$ event was detected by SMEI $\sim 15$ hours before it reached Earth causing a major geomagnetic storm. LASCO observed two ejections that likely merged to become a single halo viewed by SMEI. Two other Earth-directed CMEs were observed by SMEI during the burst of extreme solar activity in late October 2003. In addition to its primary goal of detecting and tracking ICMEs, the SMEI data will also serve as a testbed for the Heliospheric Imagers on the STEREO mission and may provide all-sky context observations for that and other upcoming NASA missions, particularly Solar-B and the Solar Dynamics Observatory.

\section{The Importance and Geoeffectiveness of CMEs}

CMEs are responsible for the most geoeffective solar wind disturbances and, therefore, the largest storms. Enhanced solar wind speeds and southward magnetic fields associated with interplanetary shocks and ejecta are known to be important causes of storms (e.g., Tsurutani et al., 1988; Gosling et al., 1991). Since the launch of SOHO, halo CMEs have been used to study the influence of Earth-directed CMEs on geoactivity. Webb et al. (2000), Cane et al. (2000) and St. Cyr et al. (2000), Cane \& Richardson (2003) and Zhao \& Webb (2003) all found good associations between frontside halo CMEs and ejecta signatures at $1 \mathrm{AU}$, especially around solar minimum, with varying degrees of 
correspondence over other parts of the cycle. Webb et al. (2000) compared the onset times of the frontside halo events in early 1997 with moderate-level storms at Earth $2-5$ days later, finding that all 6 frontside halos with surface sources near sun center were associated with magnetic clouds at 1 AU and storms. St. Cyr et al. (2000) found similar, but weaker associations between halo CMEs and storms, concluding that $83 \%$ of intense storms were preceded by frontside halo CMEs. However, 25 of the frontside halo CMEs did not produce such large storms and were, therefore, false alarms.

All the earlier studies included partial halo CMEs but by 2000, LASCO had observed a sufficient number of CMEs to permit statistical studies using only full $\left(360^{\circ}\right)$ halo events, those most likely to be directed along the Sun-Earth line. In a study of 89 frontside full halo (FFH) CMEs observed from 1996-2000, Webb (2002) found that $~ 70 \%$ of the halos were associated with shocks and/or other ejecta signatures at 1 AU. Magnetic clouds or cloud-like structures were involved with $\sim 60 \%$ of the halos $(\sim 70 \%$ if the peculiar year 1999 is excluded). However, Cane \& Richardson (2003), using data from 1996-2002, concluded that only about half of all frontside halo CMEs have associated ejecta at Earth, even if only FFH CMEs are considered.

Zhao \& Webb (2003) studied the storm effectiveness of FFH CMEs observed from 1996-2000. The fractional association between the FFH CMEs and moderate or intense storms (peak Dst $<-50 \mathrm{nT}$ ) decreased from 0.9 in 1997 to 0.4 in 1999, then increased to 0.7 at maximum in 2000. Thus, when averaged over this entire period, $65 \%$ of the FFH CMEs were associated with moderate-level or greater storms. Webb (2002) showed that the average travel time from the onsets of the halo CMEs to the onsets of the storms at Earth was 3.3 days. The average CME rate and speeds increased with the cycle and the travel time decreased, as expected for more energetic events.

Thus, we can conclude that halo CMEs with associated surface features, especially if near Sun center, usually presage at least moderate geomagnetic storms, but that at least half as many such storms occur without halo-CME forewarning. Therefore, the simple observation of the occurrence of a probable frontside halolike CME has already significantly increased our ability to forecast the occurrence of storms of moderate or greater levels at Earth. Moderate storms not associated with CMEs are usually caused by Earth passage through the heliospheric current sheet (HCS) and related corotating interaction regions (CIRs).

Why are CMEs in general and halo CMEs in particular less well associated with storms near solar maximum than minimum? There are several possible reasons. The occurrence rate of CMEs increases by an order of magnitude near maximum, leading to multiple CMEs per day over the Sun or even from a single region. Gopalswamy et al. (2001) have found evidence for interacting CMEs wherein a faster CME overtakes a slower one within $30 \mathrm{R}_{S}$ of the Sun, producing a collision or interaction. Reconnection or sandwiching of each CMEs's field lines are likely in such cases. The combination of sequential eruptions of CMEs and their subsequent interactions can produce complex ejecta at 1 AU. Such ejecta often consist of high speed flows with shocks and other ICME signatures, but poorly defined magnetic structures, or 'tangled fields' (Burlaga et al., 2001). In addition, the rate at which CMEs actually encounter Earth near maximum is modified by their broadening latitude distribution. Thus, although the CME rate is considerably higher at maximum, proportionally fewer CMEs are ejected near the ecliptic because of the highly tilted streamer belt. Because the flux ropes associated with this tilted belt tend to be north-south oriented, the individual CMEs that do reach the ecliptic may contain little southward field. Finally, the "background" solar wind into which the CMEs are injected is itself much more complex near maximum. This creates more frequent and complicated 
interactions of ejecta with the existing structure leading to distortions and compressions which are difficult to simulate and predict.

\section{Summary and Conclusions}

The distribution of geomagnetic disturbances over the solar activity cycle tends to have two peaks representing two major components of geoactivity with different solar and heliospheric sources: one associated with transient solar activity that peaks with the sunspot cycle, and the other associated with recurrent CIRs, CMEs and high speed streams from coronal holes during the declining phase. In terms of space weather, CMEs are the most important kind of transient activity because they link the activity at the Sun and its propagation through the heliosphere to the Earth. However, we now know that both CMEs and CIRs-high speed stream ensembles can be geoeffective at all phases of the cycle.

What makes solar and heliospheric disturbances geoeffective, in the sense of causing storms, is primarily due to southward IMF and compression. Southward IMF is important because it allows merging of the IMF and Earth's magnetic field and transfer of solar wind energy and mass into the magnetosphere. Compression is important because it strengthens existing southward IMF and, to a lesser extent, increases density. CMEs, the most geoeffective structures, usually contain long-duration flows of southward IMF and fast CMEs compress any southward IMF at their leading edges and behind shocks created by the speed difference. In addition, CMEs themselves can carry high-density structures, such as solar filaments. High-speed streams are geoeffective if they compress any southward IMF in CIRs. This compression can be enhanced when CIRs interact with CMEs erupting through the HCS.

The SOHO-era of CME observations has provided new insights into halo CMEs and the solar cycle variation of CMEs in general. Future analyses of these rich data sets will improve our understanding of the role CMEs play in space weather throughout the solar cycle.

\section{Acknowledgements}

I thank the Organizing Committee of the IAU 223 Symposium for inviting my presentation at the meeting. I benefitted from data from the SOHO mission, which is an international collaboration between NASA and ESA, and also from the SOHO/ LASCO CME catalog, generated and maintained by the Center for Solar Physics and Space Weather, The Catholic University of America in cooperation with the Naval Research Laboratory and NASA. I am grateful to N. Gopalswamy for use of his group's CME results. This work was supported at Boston College by Air Force contract AF19628-00-K-0073 and NASA grant NAG5-10833.

\section{References}

Antiochos S.K., C.R. DeVore \& J.A. Klimchuk 1999 Astrophys. J. 510, 485-493.

Biesecker, D.A., Myers, D.C., Thompson, B.J., Hammer, D.M., \& Vourlidas, A. 2002 Astrophys. J. 569, 1009-1015.

Brueckner, G.E. et al. 1995 Solar Phys. 162, 357-402.

Burlaga L.F., R.M. Skoug, C.W. Smith, D.F. Webb, T.H. Zurbuchen and A. Reinard, J. 2001 Geophys. Res. 106, 20,957-20,977.

Burkepile, J.T., Hundhausen, A.J., Stanger, A.L., St. Cyr, O.C., and Seiden, J.A., 2004 J. Geophys. Res. 109, A03103, doi: 10.1029/2003JA010149.

Cane, H.V. \& Richardson, I.G. 2003 J. Geophys. Res. 108, 1156, doi:10.1029/2002JA009817.

Cane H.V., Richardson, I.G. \& St. Cyr, O.C. 2000 Geophys. Res. Lett. 27, 3591-3594.

Canfield, R.F., Hudson, H.S. \& McKenzie, D.E. 1999 Geophys. Res. Lett. 26, 627. 
Cliver, E.W. \& Hudson, H.S. 2002 J. Atmos. Sol. Terr. Phys. 64, 231-252.

Eyles, C.J. et al. 2003 Solar Phys. 217, 319347.

Gopalswamy, N., S. 2004 in The Sun and the Heliosphere as an Integrated system ASSL series, ed. G. Poletto and S. Suess, Kluwer, in press.

Gopalswamy, N.S., Nunes, S., Yashiro, S. \& Howard, R.A. 2003 Adv. Space Res. in press.

Gosling, J.T. 1993 J. Geophys. Res. 98, 18,937-18,949.

Gosling, J.T., D.J. McComas, JL. Phillips, \& S.J. Bame 1991 J. Geophys. Res. 96, 7831-7839.

Harrison, R.A. 1986 Astron. Astrophys. 162, 283-291.

Howard, R.A., D.J. Michels, N.R. Sheeley, Jr., and M.J. Koomen 1982 Astrophys. J. 263, L101L104.

Howard, R.A., Sheeley, N.R. Jr., Koomen, M.J., and Michels, D.J. 1985 J. Geophys. Res. 90, 8173-8191.

Howard, R.A., Sheeley, N.R. Jr., Michels, D.J. and Koomen, M.J. 1986 in The Sun and the Heliosphere in Three Dimensions, ed., R. G. Marsden, D. Reidel, Dordrecht, Holland, 107111.

Hudson, H.S. 1990 Sol. Phys. 133, 357.

Hudson, H.S. \& Cliver, E.W. 2001 J. Geophys. Res. 106, 25,199-25,213.

Hudson, H.S. \& Webb, D.F. 1997 in Coronal Mass Ejections, eds., N. Crooker et al. GM 99, Washington, D.C., AGU, 27.

Hundhausen, A.J. 1993 J. Geophys. Res. 98, 13,177-13,200.

Hundhausen, A.J., Burkepile, J.T. \& St. Cyr, O.C. 1994 J. Geophys. Res. 99, 6543-6552.

Jackson, B.V. 1992 in Eruptive Solar Flares, Lecture Notes in Physics 399, Springer-Verlag, Berlin, 248257.

Kahler, S.W 1977 Astrophys. J. 214, 891-897.

Kahler, S.W. 1992 Annu. Rev. Astron. Astrophys. 30, 113.

Plunkett, S.P. et al. 2002 Adv. Space Res. 29, 1473-1488.

Richardson, I.G., E.W. Cliver, \& H.V. Cane 2000 J. Geophys. Res.105, 18,203.

Richardson, I.G., E.W. Cliver, \& H.V. Cane 2001 Geophys. Res. Lett. 28, 2569-2572.

Sheeley, N.R., Jr., Howard, R.A., Koomen, M.J., \& Michels, D.M. 1983 Astrophys. J. 272, 349-354.

Sterling, A.C., Hudson, H.S., Thompson, B.J. \& Zarro, D.M. 2000 Astrophys. J. 532, 628-647.

St. Cyr, O.C. et al. 2000 J. Geophys. Res. 105, 18,169-18,185.

Tappin et al. 2004 Geophys. Res. Lett. 31, L02802, doi:10.1029/2003GL018766.

Thompson, B.J., E.W. Cliver, N. Nitta, C. Delannee \& J.P. Delaboudiniere 2000 Geophys. Res. Lett. 27, 1431-1434.

Tripathi, D., Bothmer, V. \& Cremades, H. 2004 Astron. \&3 Astrophys. 422, 337-349.

Tsurutani, B.T., W.D. Gonzalez, F. Tang, S.I. Akasofu, \& E.J. Smith 1988 J. Geophys. Res. 93, 8519-8531.

von Steiger, R. \& Zurbuchen, T.H. 2003 in Solar Variability as an Input to the Earth's Environment, ESA SP 535, ed., A Wilson, ESTEC, Noordwijk, The Netherlands, 835-840.

Vourilidas, A.P. Subramanian, K.P. Dere, \& R.A. Howard 2000 Astrophys. J. 534, 456-467.

Vourilidas, A., Buzasi, D., Howard, R.A., \& Esfandiari, E. 2002 in Solar Variability: From Core to Outer Frontiers, ESA SP 506, ed., A.Wilson, ESTEC, Noordwijk, The Netherlands, 91-94.

Wang, Y.-M., J. Lean, \& N.R. Sheeley, Jr. 2000 Geophys. Res. Lett. 27, 505.

Webb, D.F. 2002 in From Solar Min to Max: Half a Solar Cycle with SOHO, ESA SP 508, ed., A.Wilson, ESTEC, Noordwijk, The Netherlands, 409-419.

Webb, D.F. \& Howard, R.A. 1994 J. Geophys. Res. 99, 4201.

Webb, D.F., R.A. Howard, and B.V. Jackson 1996 in Solar Wind Eight eds., D. Winterhalter et al. AIP Conf. Proc. 382. Woodbury, N.Y., Amer. Inst. of Phys., 540-543.

Webb, D., S. Kahler, P.McIntosh, \& J. Klimchuk 1997 J. Geophys. Res. 102, 24,161-24,174.

Webb, D.F., E.W. Cliver, N.U. Crooker, O.C. St. Cyr \& B.J. Thompson 2000 J. Geophys. Res. 105, 7491-7508.

Yashiro, S., Gopalswamy, N., Michalek, G., St. Cyr, O.C., Plunkett, S.P., Rich, N.B., \& Howard, R.A. 2004 J. Geophys. Res. 109, A07105, doi:10.1029/2003JA010282.

Zhao, X.P. \& Webb, D.F. 2003 J. Geophys. Res. 108, 1234, doi:10.1029/2002JA009606. 